\title{
Stark broadening of several Bi IV spectral lines of astrophysical interest
}

\author{
C. Colón, ${ }^{\star}$ C. Moreno-Díaz, I. de Andrés-García and A. Alonso-Medina \\ Física Aplicada, E.T.S.I.D. Industrial, Universidad Politécnica de Madrid, Ronda de Valencia 3, E-28012 Madrid, Spain
}

Accepted 2017 May 24. Received 2017 May 20; in original form 2017 May 11

\begin{abstract}
The presence of spectral lines of bismuth in stellar atmospheres has been reported in different stars. The anomalous values of the spectral intensities of $\mathrm{Bi}$ II and $\mathrm{Bi}$ III, compared to the theoretical Local Termodinamic Equilibrium (LTE) standards of $\mathrm{Bi}$ I/ $\mathrm{Bi}$ iI/ $\mathrm{Bi}$ III, have been reported in the spectra obtained with the High Resolution Spectrograph of the Hubble/Goddard Space Telescope in the chemically peculiar stars HgMn stars $\chi$ Lupi and HR 7775. Spectral lines of 1436.8, 1902.3, 2630.9 and $2936.7 \AA$ of $\mathrm{Bi}$ II and 1423.4 $\AA$ of $\mathrm{Bi}$ III were reported and their relative intensities were measured in these studies Litzén \& Wahlgren 2002. These lines are overlapped with spectral lines of 1437.65, 2630.1 and $2937.1 \AA$ of Bi Iv. A study of the Stark broadening parameters of Bi IV spectral lines can help to study these overlaps. In this paper, using the Griem semi-empirical approach, we report calculated values of the Stark parameters for 64 spectral lines of $\mathrm{Bi}$ iv. The matrix elements used in these calculations have been determined from 17 configurations of Bi Iv. They were calculated using the cowAN code including core polarization effects. Data are displayed for an electron density of $10^{17} \mathrm{~cm}^{-3}$ and temperatures $T=10000-160000 \mathrm{~K}$. Also calculated radiative lifetimes for 12 levels with experimental lifetime are presented, in order to test the goodness of our calculations. Theoretical trends of the Stark width and shift parameters versus the temperature for spectral lines of astrophysical interest are displayed.
\end{abstract}

Key words: atomic data - atomic processes.

\section{INTRODUCTION}

$\mathrm{Bi}$ IV is an ionic species of high interest in the industry and in the science of new materials. Bi Iv can be considered as the main dopant in the prospective materials used for the design and synthesis of new scintillators (Zorenko et al. 2009, 2014). In astrophysics, although we have not found bibliographical references of the presence of $\mathrm{Bi}$ IV in stellar spectra if there are references of the presence of Bi I, Bi II and Bi III (Wahlgren et al. 2001; Dolk, Litzén \& Wahlgren 2002). In these studies, the spectra obtained with the High Resolution Spectrograph of the Hubble/Goddard Space Telescope in the chemically peculiar stars HgMn stars $\chi$ Lupi and HR 7775 were analysed. Spectral lines of 1436.8, 1902.3, 2630.9 and $2936.7 \AA$ of $\mathrm{Bi}$ II and 1423.4 $\mathrm{A}$ of $\mathrm{Bi}$ III were identified and their relative intensities were measured. Wahlgren reported about anomalous values of the spectral intensities of $\mathrm{Bi}$ II and $\mathrm{Bi}$ III, compared to the theoretical LTE standards of $\mathrm{Bi}$ I/ $/ \mathrm{Bi}$ II/ $\mathrm{Bi}$ III. These lines are very close to the spectral lines of 1437.65, 2630.1 and 2937.1 of $\mathrm{Bi}$ IV that may interfere with the measurement of the intensities used in these studies. An analysis of the Stark broadening parameters of the Bi IV spectral lines can help to quantify these overlaps.

\footnotetext{
^E-mail: cristobal.colon@upm.es (CC); alonso.medina@upm.es (AA-M)
}

$\mathrm{Bi}$ IV is a member of the isoelectronic sequence of mercury. This ionic specie, together with $\mathrm{Pb}$ III (also in the same sequence), has generated studies of fundamental interest in atomic physics since the $30 \mathrm{~s}$ of the last century. Atomic parameters of $\mathrm{Pb}$ III were already measured and calculated by these authors. The transition probabilities of $10 \mathrm{~Pb}$ III spectral lines were measured by Colón, Alonso-Medina \& Herran-Mertínez (1999). Later, the transition probabilities of 30 lines were measured by Alonso-Medina (2010). Theoretical calculations, obtained in intermediate coupling (IC) with an ab initio relativistic Hartree-Fock (HFR) method, for the transition probabilities of $54 \mathrm{~Pb}$ III spectral lines were presented by Colón \& Alonso-Medina (2000). Later, new theoretical calculations, including core polarization (CP) effects, for the oscillator strengths of $382 \mathrm{~Pb}$ III spectral lines were performed by AlonsoMedina, Colón \& Zanón (2009). The Stark width of 10 Pb III spectral lines were measured by Alonso-Medina \& Colón (2007) and later Alonso-Medina (2011) measured the Stark width of 15 new spectral lines. Calculations of Stark broadening parameters for 122 $\mathrm{Pb}$ III spectral lines were performed by Alonso-Medina, Colón \& Zanón (2008) and later new calculations, taking into account the effects of CP, were made by Zanón, Alonso-Medina \& Colón (2010). In this work, we have done a theoretical study similar to the one performed for $\mathrm{Pb}$ III.

McLay \& Crawford (1933) did an exhaustive study in which 360 lines of Bi IV were classified. This work was collected by Moore 


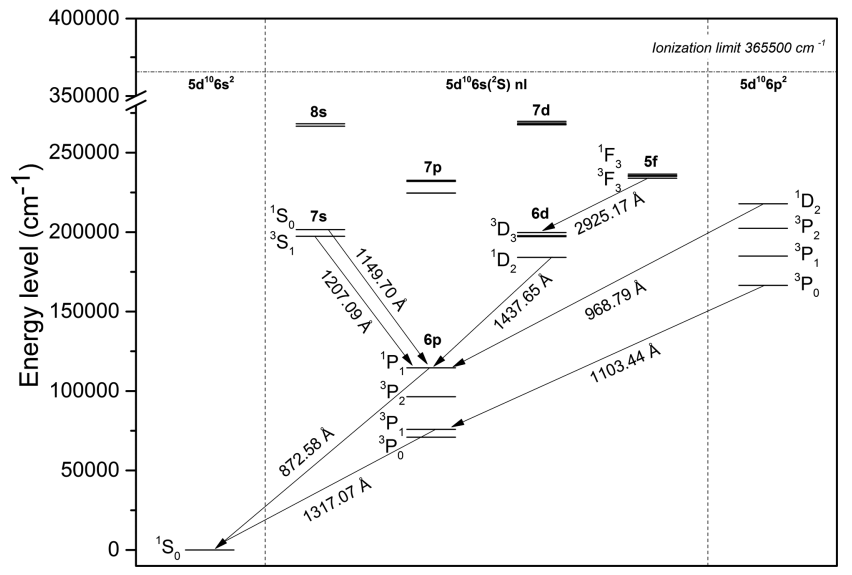

Figure 1. Partial Gotrian diagram of the Bi IV energy levels (Moore 1958), showing several spectral lines with experimental lifetime.

(1958). Calculations of energy levels of isoelectronic sequence of Hg I were made by Martin, Sugar \& Tech (1972). Later, an analysis of transitions from auto-ionization states of $\mathrm{Bi}$ IV was made by Ryabtsev, Churilov \& Joshi (2000). The 5d photoabsorption spectra of $\mathrm{Pb}$ III and $\mathrm{Bi}$ IV were studied by Banahan et al. (2008).

In the literature, we have found only several experimental values of the lifetimes. The lifetime of the $5 \mathrm{~d}^{10} 6 \mathrm{~s} 6 \mathrm{p}^{1} \mathrm{P}_{1}$ level was measured by Andersen, Kirkegård Nielsen \& Sørensen (1972). The lifetimes of nine levels of Bi IV were obtained by Pinnington et al. (1988). Later, lifetime measurements of five levels of $\mathrm{Bi}$ Iv (including the above mentioned) were made by Ansbacher et al. (1989). These values were obtained using the beam-foil technique and the "correlated analysis of decay curve' method. A summary of the existing lifetimes for the $6 s^{2}-6 s 6 p$ transitions was presented by Curtis, Irving \& Henderson (2001).

The aim of this work is to present Stark broadening parameters for several spectral lines of Bi IV. We present the first theoretical values of the Stark broadening parameters for 64 spectral lines of the $\mathrm{Bi}$ Iv that arise from configurations $5 \mathrm{~d}^{10} 6 \mathrm{sns}(n=7,8), 5 \mathrm{~d}^{10} 6 \mathrm{p}^{2}$, $5 \mathrm{~d}^{10} 6 \mathrm{snp}(n=6,7), 5 \mathrm{~d}^{9} 6 \mathrm{~s}^{2} 6 \mathrm{p}, 5 \mathrm{~d}^{10} 6 \mathrm{snd}(n=6,7)$ and $5 \mathrm{~d}^{10} 6 \mathrm{~s} 5 \mathrm{f}$. These calculations were obtained using a semiclassical approach taking into account the $\mathrm{CP}$ effects, similar to the calculations that were made by Zanón et al. (2010).

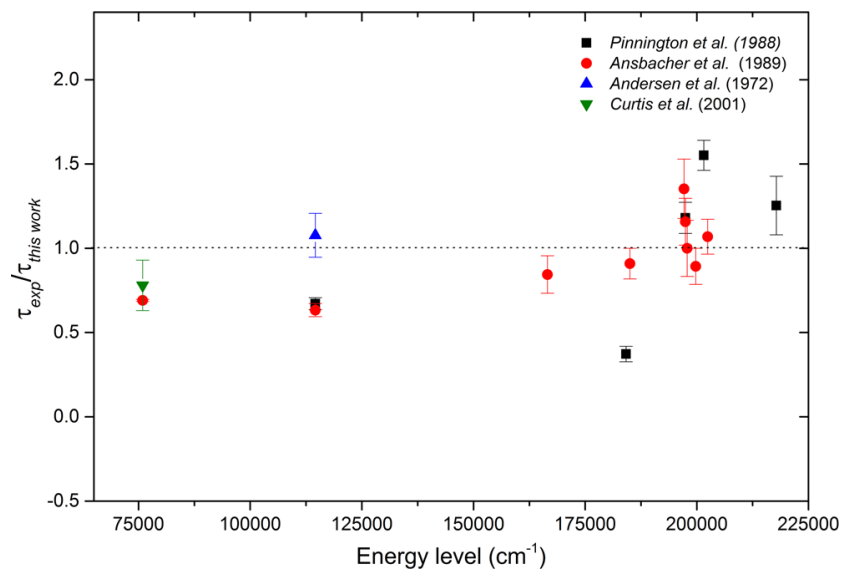

Figure 2. Ratio between experimental values of radiative lifetimes and the theoretical values obtained in this work for several levels of Bi IV $\left(\tau_{\exp } / \tau_{\text {this work }}\right)$ versus the energy of the levels.

The Stark broadening parameters are presented for a set of temperatures ranging from 5000 to $100000 \mathrm{~K}$ and an electronic density of $10^{17} \mathrm{~cm}^{-3}$. In Section 2, the theoretical calculations are presented. In Section 3, we describe our results, including the regularity of the Stark broadening parameters versus the temperature and a comparison of measured and calculated Stark width of $\mathrm{Bi}$ IV with the $\mathrm{Pb}$ III (same isoelectronic sequence).

\section{THEORETICAL CALCULATIONS}

Our calculation procedure is similar as presented by the authors in previous works, as for example: Alonso-Medina \& Herrán (1996), Colón \& Alonso-Medina (2002), Alonso-Medina et al. (2010), Colón et al. (2013), Alonso-Medina \& Colón (2014), Moreno-Díaz et al. (2014) and recently de Andrés-García, Alonso-Medina \& Colón (2016). We have used the semi-empirical formulas 23 and 35 obtained by Griem (1968), where it was taken into account the Baranger's (1958) original formulation with semi-empirical effective Gaunt factors as proposed by Van Regemorter (1962) and Seaton (1962). As it is well known (Griem 1968), a factor larger than 1.5 between calculated and experimental values can be expected with the use of this effective Gaunt factor.

Table 1. Radiative lifetimes calculated of several levels of the Bi Iv.

\begin{tabular}{lcccc}
\hline Level & \multicolumn{3}{c}{$\begin{array}{c}\text { Radiative lifetimes } \tau(\mathrm{ns}) \\
\text { Others authors }\end{array}$} \\
\hline $5 \mathrm{~d}^{10} 6 \mathrm{~s}\left({ }^{2} \mathrm{~S}\right) 7 \mathrm{~s}^{3} \mathrm{~S}_{1}$ & 0.216 & $0.255 \pm 0.020^{a}$ & $0.25 \pm 0.03^{b}$ & \\
$5 \mathrm{~d}^{10} 6 \mathrm{~s}\left({ }^{2} \mathrm{~S}\right) 7 \mathrm{~s}{ }^{1} \mathrm{~S}_{0}$ & 0.292 & $0.453 \pm 0.026^{a}$ & $0.38 \pm 0.05^{b}$ & \\
$5 \mathrm{~d}^{10} 6 \mathrm{p}^{2} \mathrm{P}_{0}$ & 0.45 & & $0.30 \pm 0.03^{b}$ & \\
$5 \mathrm{~d}^{10} 6 \mathrm{p}^{2} \mathrm{P}_{1}$ & 0.33 & & $0.31 \pm 0.03^{b}$ & \\
$5 \mathrm{~d}^{10} 6 \mathrm{p}^{2}{ }^{3} \mathrm{P}_{2}$ & 0.29 & & & \\
$5 \mathrm{~d}^{10} 6 \mathrm{p}^{2}{ }^{1} \mathrm{D}_{2}$ & 0.138 & $0.173 \pm 0.024^{a}$ & $0.00 \pm 0.07^{b}$ & \\
$5 \mathrm{~d}^{10} 6 \mathrm{~s}\left({ }^{2} \mathrm{~S}\right) 6 \mathrm{p}{ }^{3} \mathrm{P}_{1}$ & 11.57 & & $0.229 \pm 0.014^{b}$ & $0.39 \pm 0.08^{d}$ \\
$5 \mathrm{~d}^{10} 6 \mathrm{~s}\left({ }^{2} \mathrm{~S}\right) 6 \mathrm{p}{ }^{1} \mathrm{P}_{1}^{\mathrm{o}}$ & 0.362 & $0.243 \pm 0.013^{a}$ & & \\
$5 \mathrm{~d}^{10} 6 \mathrm{~s}\left({ }^{2} \mathrm{~S}\right) 6 \mathrm{~d}^{1} \mathrm{D}_{2}$ & 2.73 & $1.02 \pm 0.11^{a}$ & $0.23 \pm 0.03^{b}$ & \\
$5 \mathrm{~d}^{10} 6 \mathrm{~s}\left({ }^{2} \mathrm{~S}\right) 6 \mathrm{~d}^{3} \mathrm{D}_{1}$ & 0.17 & & $0.18 \pm 0.03^{b}$ & \\
$5 \mathrm{~d}^{10} 6 \mathrm{~s}\left({ }^{2} \mathrm{~S}\right) 6 \mathrm{~d}^{3} \mathrm{D}_{2}$ & 0.18 & & $0.25 \pm 0.03^{b}$ & \\
$5 \mathrm{~d}^{10} 6 \mathrm{~s}\left({ }^{2} \mathrm{~S}\right) 6 \mathrm{~d}^{3} \mathrm{D}_{3}$ & 0.28 & & & \\
\hline
\end{tabular}

\footnotetext{
${ }^{a}$ Ansbacher et al. (1989).

${ }^{b}$ Pinnington et al. (1988).

${ }^{c}$ Curtis et al. (2001).

${ }^{d}$ Andersen et al. (1972).
} 
Table 2. Bi iv $5 \mathrm{~d}^{10} 6 \mathrm{~s}\left({ }^{2} \mathrm{~S}\right) \mathrm{ns}(n=7-8)$ line widths (FWHM), $\omega(\mathrm{pm})$, and shifts, $d(\mathrm{pm})$, normalized to $N e=10^{17} \mathrm{~cm}^{-3}$.

\begin{tabular}{|c|c|c|c|c|c|}
\hline Transition array & Multiplet & $\lambda(\AA)^{a}$ & $T\left(10^{4} \mathrm{~K}\right)$ & $\omega(\mathrm{pm})$ & $d(\mathrm{pm})$ \\
\hline \multirow[t]{6}{*}{$5 d^{10} 6 s\left({ }^{2} S\right) 6 p-5 d^{10} 6 s\left({ }^{2} S\right) 7 s$} & \multirow[t]{6}{*}{${ }^{3} \mathrm{P}_{0}^{\mathrm{o}}-{ }^{3} \mathrm{~S}_{1}$} & \multirow[t]{6}{*}{790.62} & 1 & 1.55 & -1.26 \\
\hline & & & 2 & 1.10 & -0.88 \\
\hline & & & 3 & 0.64 & -0.47 \\
\hline & & & 5 & 0.54 & -0.41 \\
\hline & & & 10 & 0.74 & -0.70 \\
\hline & & & 16 & 0.78 & -0.73 \\
\hline \multirow[t]{6}{*}{$5 d^{10} 6 s\left({ }^{2} S\right) 6 p-5 d^{10} 6 s\left({ }^{2} S\right) 7 s$} & \multirow{6}{*}{${ }^{3} \mathrm{P}_{1}^{\mathrm{o}}-{ }^{3} \mathrm{~S}_{1}$} & \multirow[t]{6}{*}{822.91} & 1 & 2.00 & -1.66 \\
\hline & & & 2 & 1.41 & -1.17 \\
\hline & & & 3 & 0.88 & -0.68 \\
\hline & & & 5 & 0.73 & -0.57 \\
\hline & & & 10 & 0.89 & -0.84 \\
\hline & & & 16 & 0.88 & -0.83 \\
\hline \multirow[t]{6}{*}{$5 d^{10} 6 s\left({ }^{2} S\right) 6 p-5 d^{10} 6 s\left({ }^{2} S\right) 7 s$} & \multirow{6}{*}{${ }^{3} \mathrm{P}_{2}^{\mathrm{o}}-{ }^{3} \mathrm{~S}_{1}$} & \multirow[t]{6}{*}{989.87} & 1 & 3.27 & -2.80 \\
\hline & & & 2 & 2.31 & -1.98 \\
\hline & & & 3 & 1.49 & -1.22 \\
\hline & & & 5 & 1.22 & -1.01 \\
\hline & & & 10 & 1.19 & -1.12 \\
\hline & & & 16 & 1.38 & -1.31 \\
\hline \multirow[t]{6}{*}{$5 d^{10} 6 s\left({ }^{2} S\right) 6 p-5 d^{10} 6 s\left({ }^{2} S\right) 7 s$} & \multirow{6}{*}{${ }^{1} \mathrm{P}_{1}^{\mathrm{o}}-{ }^{3} \mathrm{~S}_{1}$} & \multirow[t]{6}{*}{$1207.09^{*}$} & 1 & 4.77 & -3.73 \\
\hline & & & 2 & 3.37 & -2.63 \\
\hline & & & 3 & 2.16 & -1.55 \\
\hline & & & 5 & 1.77 & -1.31 \\
\hline & & & 10 & 2.03 & -1.82 \\
\hline & & & 16 & 2.02 & -1.87 \\
\hline \multirow[t]{6}{*}{$5 d^{10} 6 s\left({ }^{2} S\right) 6 p-5 d^{10} 6 s\left({ }^{2} S\right) 7 s$} & \multirow[t]{6}{*}{${ }^{3} \mathrm{P}_{1}^{\mathrm{o}}-{ }^{1} \mathrm{~S}_{0}$} & \multirow[t]{6}{*}{795.83} & 1 & 0.93 & -0.80 \\
\hline & & & 2 & 0.66 & -0.56 \\
\hline & & & 3 & 0.40 & -0.32 \\
\hline & & & 5 & 0.41 & -0.35 \\
\hline & & & 10 & 0.40 & -0.38 \\
\hline & & & 16 & 0.35 & -0.32 \\
\hline \multirow[t]{9}{*}{$5 d^{10} 6 s\left({ }^{2} S\right) 6 p-5 d^{10} 6 s\left({ }^{2} S\right) 7 s$} & \multirow[t]{9}{*}{${ }^{1} \mathrm{P}_{1}^{\mathrm{o}}-{ }^{1} \mathrm{~S}_{0}$} & 1149.7 & 0.5 & 3.34 & -2.56 \\
\hline & & & 1 & 2.36 & -1.81 \\
\hline & & & 2 & 1.67 & -1.28 \\
\hline & & & 2.5 & 1.30 & -0.95 \\
\hline & & & 3 & 1.07 & -0.75 \\
\hline & & & 5 & 1.03 & -0.79 \\
\hline & & & 7.5 & 0.98 & -0.79 \\
\hline & & & 10 & 0.93 & -0.80 \\
\hline & & & 16 & 0.82 & -0.73 \\
\hline $5 d^{10} 6 s\left({ }^{2} S\right) 7 p-5 d^{10} 6 s\left({ }^{2} S\right) 8 s$ & ${ }^{3} \mathrm{P}_{1}^{\mathrm{o}}-{ }^{3} \mathrm{~S}_{1}$ & 2376.76 & 1 & 25.66 & 4.37 \\
\hline & & & 2 & 18.79 & 3.73 \\
\hline & & & 3 & 13.03 & 0.73 \\
\hline & & & 5 & 7.16 & 4.43 \\
\hline & & & 10 & 12.38 & 4.58 \\
\hline & & & 16 & 13.86 & 4.25 \\
\hline $5 d^{10} 6 s\left({ }^{2} S\right) 7 p-5 d^{10} 6 s\left({ }^{2} S\right) 8 s$ & ${ }^{3} \mathrm{P}_{0}^{\mathrm{o}}-{ }^{3} \mathrm{~S}_{1}$ & 2377.16 & 1 & 13.37 & 5.72 \\
\hline & & & 2 & 9.45 & 4.04 \\
\hline & & & 3 & 6.55 & 2.13 \\
\hline & & & 5 & 2.97 & 1.88 \\
\hline & & & 10 & 6.01 & 3.09 \\
\hline & & & 16 & 6.89 & 3.34 \\
\hline $5 d^{10} 6 s\left({ }^{2} S\right) 7 p-5 d^{10} 6 s\left({ }^{2} S\right) 8 s$ & ${ }^{3} \mathrm{P}_{2}^{\mathrm{o}}-{ }^{3} \mathrm{~S}_{1}$ & 2937.12 & 1 & 14.72 & 10.92 \\
\hline & & & 2 & 10.40 & 7.72 \\
\hline & & & 3 & 8.49 & 6.30 \\
\hline & & & 5 & 3.19 & 2.14 \\
\hline & & & 10 & 6.59 & 4.77 \\
\hline & & & 16 & 7.55 & 5.50 \\
\hline
\end{tabular}

Notes. ${ }^{*}$ A positive shift is red.

${ }^{a}$ Moore (1958). 
Table 3. Bi iv $5 \mathrm{~d}^{10} 6 \mathrm{p}^{2}, 5 \mathrm{~d}^{10} 6 \mathrm{~s}\left({ }^{2} \mathrm{~S}\right) \mathrm{np}(n=6-7)$ and $5 \mathrm{~d}^{9} 6 \mathrm{~s}^{2} 6 \mathrm{p}$ line widths (FWHM), $\omega$ (pm), and shifts, $d(\mathrm{pm})$, normalized to $\mathrm{Ne}=10^{17} \mathrm{~cm}^{-3}$.

\begin{tabular}{|c|c|c|c|c|c|}
\hline Transition array & Multiplet & $\lambda(\AA)^{a}$ & $T\left(10^{4} \mathrm{~K}\right)$ & $\omega(\mathrm{pm})$ & $d(\mathrm{pm})$ \\
\hline \multirow[t]{6}{*}{$5 d^{10} 6 s\left({ }^{2} S\right) 6 p-5 d^{10} 6 p^{2}$} & ${ }^{3} \mathrm{P}_{1}^{\mathrm{o}}-{ }^{3} \mathrm{P}_{0}$ & 1103.44 & 1 & 0.91 & -0.73 \\
\hline & & & 2 & 0.65 & -0.51 \\
\hline & & & 3 & 0.53 & -0.42 \\
\hline & & & 5 & 0.41 & -0.33 \\
\hline & & & 10 & 0.25 & -0.23 \\
\hline & & & 16 & 0.12 & -0.08 \\
\hline \multirow[t]{6}{*}{$5 d^{10} 6 s\left({ }^{2} S\right) 6 p-5 d^{10} 6 p^{2}$} & ${ }^{1} \mathrm{P}_{1}^{\mathrm{o}}-{ }^{3} \mathrm{P}_{0}$ & $1924.93^{*}$ & 1 & 3.97 & -2.61 \\
\hline & & & 2 & 2.80 & -1.84 \\
\hline & & & 3 & 2.29 & -1.50 \\
\hline & & & 5 & 1.77 & -1.17 \\
\hline & & & 10 & 1.04 & -0.73 \\
\hline & & & 16 & 0.66 & -0.44 \\
\hline \multirow{6}{*}{$5 d^{10} 6 s\left({ }^{2} S\right) 6 p-5 d^{10} 6 p^{2}$} & ${ }^{3} \mathrm{P}_{0}^{\mathrm{o}}-{ }^{3} \mathrm{P}_{1}$ & 876.80 & 1 & 0.30 & -0.03 \\
\hline & & & 2 & 0.22 & -0.02 \\
\hline & & & 3 & 0.18 & -0.02 \\
\hline & & & 5 & 0.14 & -0.01 \\
\hline & & & 10 & 0.08 & -0.02 \\
\hline & & & 16 & 0.04 & 0.01 \\
\hline \multirow[t]{6}{*}{$5 \mathrm{~d}^{10} 6 \mathrm{~s}\left({ }^{2} \mathrm{~S}\right) 6 \mathrm{p}-5 \mathrm{~d}^{10} 6 \mathrm{p}^{2}$} & ${ }^{3} \mathrm{P}_{1}^{\mathrm{o}}-{ }^{3} \mathrm{P}_{1}$ & 916.69 & 1 & 0.73 & -0.40 \\
\hline & & & 2 & 0.51 & -0.28 \\
\hline & & & 3 & 0.42 & -0.23 \\
\hline & & & 5 & 0.33 & -0.18 \\
\hline & & & 10 & 0.20 & -0.13 \\
\hline & & & 16 & 0.10 & -0.04 \\
\hline \multirow[t]{6}{*}{$5 \mathrm{~d}^{10} 6 \mathrm{~s}\left({ }^{2} \mathrm{~S}\right) 6 \mathrm{p}-5 \mathrm{~d}^{10} 6 \mathrm{p}^{2}$} & ${ }^{3} \mathrm{P}_{2}^{\mathrm{o}}-{ }^{3} \mathrm{P}_{1}$ & 1128.78 & 1 & 1.59 & -1.13 \\
\hline & & & 2 & 1.13 & -0.80 \\
\hline & & & 3 & 0.92 & -0.65 \\
\hline & & & 5 & 0.71 & -0.51 \\
\hline & & & 10 & 0.17 & -0.08 \\
\hline & & & 16 & 0.28 & -0.20 \\
\hline \multirow[t]{6}{*}{$5 d^{10} 6 s\left({ }^{2} S\right) 6 p-5 d^{10} 6 p^{2}$} & ${ }^{1} \mathrm{P}_{1}^{\mathrm{o}}-{ }^{3} \mathrm{P}_{1}$ & $1420.21^{*}$ & 1 & 2.39 & -1.18 \\
\hline & & & 2 & 1.69 & -0.83 \\
\hline & & & 3 & 1.38 & -0.68 \\
\hline & & & 5 & 1.07 & -0.53 \\
\hline & & & 10 & 0.64 & -0.33 \\
\hline & & & 16 & 0.39 & -0.20 \\
\hline \multirow[t]{6}{*}{$5 d^{10} 6 s\left({ }^{2} s\right) 6 p-5 d^{10} 6 p^{2}$} & ${ }^{3} \mathrm{P}_{1}^{\mathrm{o}}-{ }^{3} \mathrm{P}_{2}$ & 790.46 & 1 & 1.03 & -0.62 \\
\hline & & & 2 & 0.73 & -0.44 \\
\hline & & & 3 & 0.55 & -0.31 \\
\hline & & & 5 & 0.42 & -0.24 \\
\hline & & & 10 & 0.38 & -0.28 \\
\hline & & & 16 & 0.32 & -0.25 \\
\hline \multirow[t]{6}{*}{$5 \mathrm{~d}^{10} 6 \mathrm{~s}\left({ }^{2} \mathrm{~S}\right) 6 \mathrm{p}-5 \mathrm{~d}^{10} 6 \mathrm{p}^{2}$} & ${ }^{3} \mathrm{P}_{2}^{\mathrm{o}}-{ }^{3} \mathrm{P}_{2}$ & 943.30 & 1 & 1.81 & -1.25 \\
\hline & & & 2 & 1.28 & -0.89 \\
\hline & & & 3 & 0.97 & -0.65 \\
\hline & & & 5 & 0.75 & -0.50 \\
\hline & & & 10 & 0.46 & -0.31 \\
\hline & & & 16 & 0.54 & -0.45 \\
\hline \multirow[t]{6}{*}{$5 \mathrm{~d}^{10} 6 \mathrm{~s}\left({ }^{2} \mathrm{~S}\right) 6 \mathrm{p}-5 \mathrm{~d}^{10} 6 \mathrm{p}^{2}$} & ${ }^{1} \mathrm{P}_{1}^{\mathrm{o}}-{ }^{3} \mathrm{P}_{2}$ & 1138.54 & 1 & 1.30 & -0.99 \\
\hline & & & 2 & 0.92 & -0.70 \\
\hline & & & 3 & 0.75 & -0.57 \\
\hline & & & 5 & 0.58 & -0.44 \\
\hline & & & 10 & 0.36 & -0.26 \\
\hline & & & 16 & 0.21 & -0.17 \\
\hline \multirow[t]{6}{*}{$5 \mathrm{~d}^{10} 6 \mathrm{~s}\left({ }^{2} \mathrm{~S}\right) 6 \mathrm{p}-5 \mathrm{~d}^{10} 6 \mathrm{p}^{2}$} & ${ }^{3} \mathrm{P}_{1}^{\mathrm{o}}-{ }^{1} \mathrm{D}_{2}$ & $704.74^{*}$ & 1 & 1.24 & -0.61 \\
\hline & & & 2 & 0.90 & -0.45 \\
\hline & & & 3 & 0.90 & -0.54 \\
\hline & & & 5 & 0.87 & -0.58 \\
\hline & & & 10 & 0.76 & -0.56 \\
\hline & & & 16 & 0.64 & -0.54 \\
\hline \multirow[t]{3}{*}{$5 \mathrm{~d}^{10} 6 \mathrm{~s}\left({ }^{2} \mathrm{~S}\right) 6 \mathrm{p}-5 \mathrm{~d}^{10} 6 \mathrm{p}^{2}$} & ${ }^{3} \mathrm{P}_{2}^{\mathrm{o}}-{ }^{1} \mathrm{D}_{2}$ & $823.72^{*}$ & 1 & 1.95 & -1.11 \\
\hline & & & 2 & 1.41 & -0.82 \\
\hline & & & 3 & 1.38 & -0.90 \\
\hline
\end{tabular}


Table 3 - continued

\begin{tabular}{|c|c|c|c|c|c|}
\hline Transition array & Multiplet & $\lambda(\AA)^{a}$ & $T\left(10^{4} \mathrm{~K}\right)$ & $\omega(\mathrm{pm})$ & $d(\mathrm{pm})$ \\
\hline & & & 5 & 1.30 & -0.92 \\
\hline & & & 10 & 0.97 & -0.70 \\
\hline & & & 16 & 0.94 & -0.81 \\
\hline \multirow[t]{6}{*}{$5 d^{10} 6 s\left({ }^{2} S\right) 6 p-5 d^{10} 6 p^{2}$} & ${ }^{1} \mathrm{P}_{1}^{\mathrm{o}}-{ }^{1} \mathrm{D}_{2}$ & 968.79 & 1 & 2.64 & -1.25 \\
\hline & & & 2 & 1.91 & -0.93 \\
\hline & & & 3 & 1.88 & -1.08 \\
\hline & & & 5 & 1.77 & -1.15 \\
\hline & & & 10 & 1.51 & -1.07 \\
\hline & & & 16 & 1.28 & -1.07 \\
\hline \multirow[t]{9}{*}{$5 d^{10} 6 s^{2}-5 d^{10} 6 s\left({ }^{2} S\right) 6 p$} & ${ }^{1} \mathrm{~S}_{0}-{ }^{3} \mathrm{P}_{1}^{\mathrm{o}}$ & 1317.07 & 0.5 & 1.68 & -1.61 \\
\hline & & & 1 & 1.19 & -1.14 \\
\hline & & & 2 & 0.84 & -0.81 \\
\hline & & & 2.5 & 0.75 & -0.72 \\
\hline & & & 3 & 0.69 & -0.66 \\
\hline & & & 5 & 0.53 & -0.51 \\
\hline & & & 7.5 & 0.43 & -0.44 \\
\hline & & & 10 & 0.34 & -0.34 \\
\hline & & & 16 & 0.15 & -0.14 \\
\hline \multirow[t]{9}{*}{$5 d^{10} 6 s^{2}-5 d^{10} 6 s\left({ }^{2} S\right) 6 p$} & ${ }^{1} \mathrm{~S}_{0}-{ }^{1} \mathrm{P}_{1}^{\mathrm{o}}$ & 872.58 & 0.5 & 1.08 & -0.82 \\
\hline & & & 1 & 0.77 & -0.58 \\
\hline & & & 2 & 0.54 & -0.41 \\
\hline & & & 2.5 & 0.48 & -0.37 \\
\hline & & & 3 & 0.44 & -0.34 \\
\hline & & & 5 & 0.34 & -0.26 \\
\hline & & & 7.5 & 0.24 & -0.19 \\
\hline & & & 10 & 0.21 & -0.15 \\
\hline & & & 16 & 0.12 & -0.10 \\
\hline \multirow[t]{6}{*}{$5 d^{10} 6 s\left({ }^{2} S\right) 6 d-5 d^{10} 6 s\left({ }^{2} S\right) 7 p$} & ${ }^{3} \mathrm{D}_{1}-{ }^{3} \mathrm{P}_{0}^{\mathrm{o}}$ & 3645.16 & 1 & 33.21 & -15.85 \\
\hline & & & 2 & 23.49 & -11.21 \\
\hline & & & 3 & 14.11 & -9.57 \\
\hline & & & 5 & 9.78 & -2.59 \\
\hline & & & 10 & 15.25 & -6.73 \\
\hline & & & 16 & 16.61 & -8.71 \\
\hline \multirow[t]{7}{*}{$5 d^{10} 6 s\left({ }^{2} S\right) 7 s-5 d^{10} 6 s\left({ }^{2} S\right) 7 p$} & ${ }^{3} \mathrm{~S}_{1}-{ }^{3} \mathrm{P}_{0}^{\mathrm{o}}$ & 3684.79 & 1 & 33.94 & -16.19 \\
\hline & & & 2 & 24.00 & -11.45 \\
\hline & & & 3 & 14.41 & -9.78 \\
\hline & & & 5 & 9.99 & -2.64 \\
\hline & & & 10 & 15.59 & -6.88 \\
\hline & & & 16 & 16.98 & -8.90 \\
\hline & & & 30 & 17.77 & -9.06 \\
\hline \multirow[t]{6}{*}{$5 d^{10} 6 s\left({ }^{2} S\right) 6 d-5 d^{10} 6 s\left({ }^{2} S\right) 7 p$} & ${ }^{3} \mathrm{D}_{1}-{ }^{3} \mathrm{P}_{1}^{\mathrm{o}}$ & 3643.04 & 1 & 62.07 & -18.99 \\
\hline & & & 2 & 45.40 & -11.91 \\
\hline & & & 3 & 29.32 & -12.84 \\
\hline & & & 5 & 19.61 & 3.39 \\
\hline & & & 10 & 30.20 & 3.21 \\
\hline & & & 16 & 32.98 & 6.56 \\
\hline \multirow[t]{6}{*}{$5 d^{10} 6 s\left({ }^{2} S\right) 7 s-5 d^{10} 6 s\left({ }^{2} S\right) 7 p$} & ${ }^{3} \mathrm{~S}_{1}-{ }^{3} \mathrm{P}_{1}^{\mathrm{o}}$ & 3682.59 & 1 & 75.47 & -30.77 \\
\hline & & & 2 & 54.85 & -20.14 \\
\hline & & & 3 & 33.71 & -16.47 \\
\hline & & & 5 & 24.16 & -0.35 \\
\hline & & & 10 & 37.40 & -10.64 \\
\hline & & & 16 & 41.25 & -13.94 \\
\hline \multirow[t]{6}{*}{$5 d^{10} 6 s\left({ }^{2} S\right) 6 d-5 d^{10} 6 s\left({ }^{2} S\right) 7 p$} & ${ }^{3} \mathrm{D}_{2}-{ }^{3} \mathrm{P}_{1}^{\mathrm{o}}$ & 3735.27 & 1 & 77.7 & -28.42 \\
\hline & & & 2 & 56.38 & -18.35 \\
\hline & & & 3 & 37.35 & -17.72 \\
\hline & & & 5 & 24.99 & 0.98 \\
\hline & & & 10 & 37.31 & -8.10 \\
\hline & & & 16 & 40.70 & -12.38 \\
\hline \multirow[t]{6}{*}{$5 d^{10} 6 s\left({ }^{2} S\right) 7 s-5 d^{10} 6 s\left({ }^{2} S\right) 7 p$} & ${ }^{3} \mathrm{~S}_{0}-{ }^{3} \mathrm{P}_{1}^{\mathrm{o}}$ & 4344.09 & 1 & 76.95 & -20.36 \\
\hline & & & 2 & 56.57 & -12.24 \\
\hline & & & 3 & 34.26 & -13.52 \\
\hline & & & 5 & 25.42 & 5.21 \\
\hline & & & 10 & 39.05 & -2.71 \\
\hline & & & 16 & 43.08 & -5.69 \\
\hline
\end{tabular}


Table 3 - continued

\begin{tabular}{|c|c|c|c|c|c|}
\hline Transition array & Multiplet & $\lambda(\AA)^{a}$ & $T\left(10^{4} \mathrm{~K}\right)$ & $\omega(\mathrm{pm})$ & $d(\mathrm{pm})$ \\
\hline \multirow[t]{6}{*}{$5 d^{10} 6 s\left({ }^{2} S\right) 7 s-5 d^{10} 6 s\left({ }^{2} S\right) 7 p$} & ${ }^{3} \mathrm{~S}_{1}-{ }^{3} \mathrm{P}_{2}^{\mathrm{o}}$ & 2842.44 & 1 & 22.04 & -14.55 \\
\hline & & & 2 & 15.55 & -10.11 \\
\hline & & & 3 & 9.40 & -4.96 \\
\hline & & & 5 & 7.13 & -4.53 \\
\hline & & & 10 & 10.75 & -8.42 \\
\hline & & & 16 & 11.82 & -9.23 \\
\hline \multirow[t]{6}{*}{$5 d^{10} 6 s\left({ }^{2} S\right) 6 d-5 d^{10} 6 s\left({ }^{2} S\right) 7 p$} & ${ }^{3} \mathrm{D}_{3}-{ }^{3} \mathrm{P}_{2}^{\mathrm{o}}$ & 3043.40 & 1 & 34.12 & -20.54 \\
\hline & & & 2 & 24.08 & -14.48 \\
\hline & & & 3 & 12.55 & -4.71 \\
\hline & & & 5 & 11.14 & -6.03 \\
\hline & & & 10 & 14.38 & -12.30 \\
\hline & & & 16 & 16.90 & -13.05 \\
\hline \multirow[t]{6}{*}{$5 d^{10} 6 s\left({ }^{2} S\right) 7 s-5 d^{9} 6 s^{2} 6 p$} & ${ }^{3} S_{1}-14_{2}^{\mathrm{o}}$ & 3012.86 & 1 & 44.78 & -8.91 \\
\hline & & & 2 & 31.67 & -6.30 \\
\hline & & & 3 & 21.97 & -9.03 \\
\hline & & & 5 & 11.16 & -0.35 \\
\hline & & & 10 & 20.90 & -2.42 \\
\hline & & & 16 & 23.77 & -3.35 \\
\hline \multirow[t]{6}{*}{$5 d^{10} 6 s\left({ }^{2} S\right) 6 d-5 d^{9} 6 s^{2} 6 p$} & ${ }^{3} \mathrm{D}_{3}-14_{2}^{\mathrm{o}}$ & 3239.60 & 1 & 85.64 & -33.75 \\
\hline & & & 2 & 60.51 & -23.82 \\
\hline & & & 3 & 36.86 & -15.88 \\
\hline & & & 5 & 24.32 & -7.17 \\
\hline & & & 10 & 38.20 & -16.78 \\
\hline & & & 16 & 44.07 & -18.73 \\
\hline \multirow[t]{6}{*}{$5 d^{10} 6 s\left({ }^{2} s\right) 6 d-5 d^{9} 6 s^{2} 6 p$} & ${ }^{3} \mathrm{D}_{3}-12_{4}^{\mathrm{o}}$ & 3868.77 & 1 & 58.96 & -26.41 \\
\hline & & & 2 & 41.62 & -18.60 \\
\hline & & & 3 & 18.09 & -8.09 \\
\hline & & & 5 & 20.04 & -6.72 \\
\hline & & & 10 & 25.72 & -15.56 \\
\hline & & & 16 & 29.88 & -16.57 \\
\hline
\end{tabular}

Notes. ${ }^{*}$ A positive shift is red.

${ }^{a}$ Moore (1958).

As you can see in the above-mentioned formulas, the Stark widths, $\omega_{\text {se }}$, and Stark shifts, $d$, are obtained in frequency units. These values are proportional to the full width at half-maximum (FWHM) of the spectral line, $\omega$, in wavelength units, through the expression $\omega=\omega_{\mathrm{se}} \lambda^{2} /(\pi c)$, where $\lambda$ is the wavelength and $c$ is the light speed. Also we need a set of array elements including all relevant transitions. To obtain the required matrix elements, we do not use the Coulomb approximation. We used an intermediate coupling scheme (IC) where wavefunctions were calculated using HFR approximation. In this approach, not only the transition probabilities for the allowed lines in LS coupling were obtained, but also the socalled forbidden transitions were obtained. For the IC calculations, we used the method of least-squares fitting of these experimental energy levels by means of cowAN computer code (Cowan 1981).

The system considered is complex, with high $Z=83$, where both relativistic and correlation effects must be important. CowAN code has been modified in order to include the $\mathrm{CP}$ effects in a way similar to the works presented by Alonso-Medina et al. (2009) and Zanón et al. (2010). The CP effects are included following the suggestions of Migdalek \& Baylis (1978): the CP effects can be written as the one-particle, $V_{\mathrm{P} 1}$, and two-particle, $V_{\mathrm{P} 2}$, potential models

$$
\begin{aligned}
& V_{P 1}=-\frac{1}{2} \alpha_{\mathrm{d}} \sum_{i=1}^{n} \frac{r_{i}^{2}}{\left(r_{i}^{2}+r_{\mathrm{c}}^{2}\right)^{3}}, \\
& V_{P 2}=-\alpha_{\mathrm{d}} \sum_{i>j}^{n} \frac{\vec{r}_{i} \cdot \vec{r}_{j}}{\left[\left(r_{i}^{2}+r_{\mathrm{c}}^{2}\right)\left(r_{j}^{2}+r_{\mathrm{c}}^{2}\right)\right]^{3 / 2}},
\end{aligned}
$$

where $\alpha_{\mathrm{d}}$ is the dipole polarizability of the core and $r_{\mathrm{c}}$ is the cut-off radius chosen as a measure of the size of the ionic core.

A modification in the matrix element can be made in order to take into account the potential change. The $\left\langle P_{\mathrm{nl}}|\mathrm{r}| P_{\mathrm{n}^{\prime} \mathrm{l}^{\prime}}\right\rangle$ is replaced by

$$
\begin{gathered}
\int_{0}^{\infty} P_{n l} r\left(1-\frac{\alpha_{\mathrm{d}}}{\left(r^{2}+r_{\mathrm{c}}^{2}\right)^{3 / 2}}\right) P_{n l^{\prime}} \mathrm{d} r \\
-\frac{\alpha_{\mathrm{d}}}{r_{\mathrm{c}}^{3}} \int_{0}^{r_{\mathrm{c}}} P_{n l}(r) r P_{n l^{\prime}}(r) \mathrm{d} r,
\end{gathered}
$$

where the core penetration term suggested by Hameed (1972) has also been included. For the dipole polarizability and the cut-off radius, we use the values, $\alpha_{\mathrm{d}}=3.04$ (in au) and $r_{\mathrm{c}}=1.201$ (in au), computed by Migdalek \& Baylis (1985).

The matrix elements used in these calculations have been determined from 17 configurations of Bi Iv: $5 \mathrm{~d}^{10} 6 \mathrm{~s}^{2}, 5 \mathrm{~d}^{10} 6 \mathrm{p}^{2}, 5 \mathrm{~d}^{10} 6 \mathrm{sns}$ $(n=7-10)$ and $5 \mathrm{~d}^{10} 6$ snd $(n=6-9)$ for even parity and $5 \mathrm{~d}^{10} 6 \mathrm{snp}$ $(n=6,7), 5 \mathrm{~d}^{10} 6 \mathrm{~s} \mathrm{nf}(n=5,6), 5 \mathrm{~d}^{9} 6 \mathrm{~s}^{2} 6 \mathrm{p}$ and $5 \mathrm{~d}^{9} 6 \mathrm{~s}^{2} \mathrm{nf}(n=5,6)$ for odd parity. For the calculations, we used all the experimental levels shown in the table of Moore (1958) based on McLay \& Crawford (1933) and several auto-ionization levels of Ryabtsev et al. (2000). Fig. 1 displays a partial Gotrian energy levels scheme of the Bi IV showing several spectral lines with experimental radiative lifetime.

We have $17+28$ experimental levels, which are far fewer than the number of adjustable parameters in the cowan code. Therefore, in the calculations, a factor of 0.85 was used to decrease the values of $F k, G k$ and $R k$ of the non-experimental configurations 
Table 4. Bi Iv $5 \mathrm{~d}^{10} 6 \mathrm{~s}\left({ }^{2} \mathrm{~S}\right)$ nd $(n=6-7)$ line widths (FWHM), $\omega(\mathrm{pm})$, and shifts, $d$ (pm), normalized to $N e=10^{17} \mathrm{~cm}^{-3}$.

\begin{tabular}{|c|c|c|c|c|c|}
\hline Transition array & Multiplet & $\lambda(\AA)^{a}$ & $T\left(10^{4} \mathrm{~K}\right)$ & $\omega(\mathrm{pm})$ & $d(\mathrm{pm})$ \\
\hline \multirow[t]{7}{*}{$5 d^{10} 6 s\left({ }^{2} S\right) 6 p-5 d^{10} 6 s\left({ }^{2} S\right) 6 d$} & \multirow{7}{*}{${ }^{3} \mathrm{P}_{1}^{\mathrm{o}}-{ }^{1} \mathrm{D}_{2}$} & \multirow[t]{7}{*}{923.92} & 1 & 1.01 & -0.90 \\
\hline & & & 2 & 0.72 & -0.64 \\
\hline & & & 3 & 0.59 & -0.52 \\
\hline & & & 5 & 0.42 & -0.36 \\
\hline & & & 10 & 0.29 & -0.28 \\
\hline & & & 16 & 0.24 & -0.21 \\
\hline & & & 30 & 0.33 & -0.29 \\
\hline \multirow[t]{6}{*}{$5 \mathrm{~d}^{10} 6 \mathrm{~s}\left({ }^{2} \mathrm{~S}\right) 6 \mathrm{p}-5 \mathrm{~d}^{10} 6 \mathrm{~s}\left({ }^{2} \mathrm{~S}\right) 6 \mathrm{~d}$} & \multirow{6}{*}{${ }^{3} \mathrm{P}_{2}^{\mathrm{o}}-{ }^{1} \mathrm{D}_{2}$} & \multirow[t]{6}{*}{1139.72} & 1 & 2.04 & -1.90 \\
\hline & & & 2 & 1.44 & -1.34 \\
\hline & & & 3 & 1.18 & -1.10 \\
\hline & & & 5 & 0.86 & -0.79 \\
\hline & & & 10 & 0.32 & -0.30 \\
\hline & & & 16 & 0.49 & -0.46 \\
\hline \multirow{7}{*}{$5 d^{10} 6 s\left({ }^{2} S\right) 6 p-5 d^{10} 6 s\left({ }^{2} s\right) 6 d$} & \multirow{7}{*}{${ }^{1} \mathrm{P}_{1}^{\mathrm{o}}-{ }^{1} \mathrm{D}_{2}$} & \multirow[t]{7}{*}{$1437.65^{*}$} & 1 & 3.12 & -2.40 \\
\hline & & & 2 & 2.21 & -1.70 \\
\hline & & & 3 & 1.80 & -1.38 \\
\hline & & & 5 & 1.30 & -0.98 \\
\hline & & & 10 & 0.88 & -0.69 \\
\hline & & & 16 & 0.74 & -0.63 \\
\hline & & & 30 & 1.03 & -0.83 \\
\hline \multirow[t]{6}{*}{$5 d^{10} 6 s\left({ }^{2} S\right) 6 p-5 d^{10} 6 s\left({ }^{2} s\right) 6 d$} & \multirow{6}{*}{${ }^{3} \mathrm{P}_{0}^{\mathrm{o}}-{ }^{3} \mathrm{D}_{1}$} & \multirow[t]{6}{*}{792.47} & 1 & 1.00 & -0.73 \\
\hline & & & 2 & 0.71 & -0.52 \\
\hline & & & 3 & 0.47 & -0.31 \\
\hline & & & 5 & 0.35 & -0.23 \\
\hline & & & 10 & 0.44 & -0.36 \\
\hline & & & 16 & 0.43 & -0.40 \\
\hline \multirow[t]{6}{*}{$5 d^{10} 6 s\left({ }^{2} S\right) 6 p-5 d^{10} 6 s\left({ }^{2} s\right) 6 d$} & \multirow{6}{*}{${ }^{3} \mathrm{P}_{1}^{\mathrm{o}}-{ }^{3} \mathrm{D}_{1}$} & 824.91 & 1 & 1.41 & -1.10 \\
\hline & & & 2 & 0.99 & -0.78 \\
\hline & & & 3 & 0.69 & -0.51 \\
\hline & & & 5 & 0.52 & -0.39 \\
\hline & & & 10 & 0.57 & -0.48 \\
\hline & & & 16 & 0.51 & -0.47 \\
\hline $5 d^{10} 6 s\left({ }^{2} S\right) 6 p-5 d^{10} 6 s\left({ }^{2} S\right) 6 d$ & ${ }^{3} \mathrm{P}_{2}^{\mathrm{o}}-{ }^{3} \mathrm{D}_{1}$ & 992.77 & 1 & 2.42 & -1.99 \\
\hline & & & 2 & 1.71 & -1.41 \\
\hline & & & 3 & 1.22 & -0.98 \\
\hline & & & 5 & 0.93 & -0.74 \\
\hline & & & 10 & 0.72 & -0.59 \\
\hline & & & 16 & 0.84 & -0.79 \\
\hline $5 d^{10} 6 s\left({ }^{2} S\right) 6 p-5 d^{10} 6 s\left({ }^{2} S\right) 6 d$ & ${ }^{1} \mathrm{P}_{1}^{\mathrm{o}}-{ }^{3} \mathrm{D}_{1}$ & $1211.40^{*}$ & 1 & 3.50 & -2.52 \\
\hline & & & 2 & 2.48 & -1.78 \\
\hline & & & 3 & 1.77 & -1.20 \\
\hline & & & 5 & 1.34 & -0.90 \\
\hline & & & 10 & 1.34 & -1.04 \\
\hline & & & 16 & 1.21 & -1.10 \\
\hline $5 d^{10} 6 s\left({ }^{2} S\right) 6 p-5 d^{10} 6 s\left({ }^{2} s\right) 6 d$ & ${ }^{3} \mathrm{P}_{1}^{\mathrm{o}}-{ }^{3} \mathrm{D}_{2}$ & 820.32 & 1 & 1.99 & -1.49 \\
\hline & & & 2 & 1.40 & -1.05 \\
\hline & & & 3 & 1.00 & -0.71 \\
\hline & & & 5 & 0.73 & -0.51 \\
\hline & & & 10 & 0.83 & -0.70 \\
\hline & & & 16 & 0.79 & -0.73 \\
\hline $5 d^{10} 6 s\left({ }^{2} S\right) 6 p-5 d^{10} 6 s\left({ }^{2} S\right) 6 d$ & ${ }^{3} \mathrm{P}_{2}^{\mathrm{o}}-{ }^{3} \mathrm{D}_{2}$ & $986.13^{*}$ & 1 & 3.25 & -2.56 \\
\hline & & & 2 & 2.29 & -1.80 \\
\hline & & & 3 & 1.66 & -1.26 \\
\hline & & & 5 & 1.22 & -0.91 \\
\hline & & & 10 & 1.10 & -0.92 \\
\hline & & & 16 & 1.25 & -1.16 \\
\hline $5 d^{10} 6 s\left({ }^{2} S\right) 6 p-5 d^{10} 6 s\left({ }^{2} S\right) 6 d$ & ${ }^{1} \mathrm{P}_{1}^{\mathrm{o}}-{ }^{3} \mathrm{D}_{2}$ & $1201.53^{*}$ & 1 & 4.74 & -3.36 \\
\hline & & & 2 & 3.33 & -2.36 \\
\hline & & & 3 & 2.41 & -1.62 \\
\hline & & & 5 & 1.77 & -1.16 \\
\hline & & & 10 & 1.89 & -1.51 \\
\hline & & & 16 & 1.82 & -1.65 \\
\hline & & & 30 & 2.07 & -1.73 \\
\hline $5 d^{10} 6 s\left({ }^{2} S\right) 6 p-5 d^{10} 6 s\left({ }^{2} S\right) 6 d$ & ${ }^{3} \mathrm{P}_{2}^{\mathrm{o}}-{ }^{3} \mathrm{D}_{3}$ & 967.62 & 1 & 4.02 & -3.09 \\
\hline
\end{tabular}


Table 4 - continued

\begin{tabular}{|c|c|c|c|c|c|}
\hline Transition array & Multiplet & $\lambda(\AA)^{a}$ & $T\left(10^{4} \mathrm{~K}\right)$ & $\omega(\mathrm{pm})$ & $d(\mathrm{pm})$ \\
\hline & & & 2 & 2.84 & -2.18 \\
\hline & & & 3 & 1.60 & -1.06 \\
\hline & & & 5 & 1.47 & -1.05 \\
\hline & & & 10 & 1.34 & -1.34 \\
\hline & & & 16 & 1.65 & -1.50 \\
\hline & & & 30 & 1.84 & -1.58 \\
\hline \multirow[t]{6}{*}{$5 \mathrm{~d}^{10} 6 \mathrm{~s}\left({ }^{2} \mathrm{~S}\right) 7 \mathrm{p}-5 \mathrm{~d}^{10} 6 \mathrm{~s}\left({ }^{2} \mathrm{~S}\right) 7 \mathrm{~d}$} & ${ }^{3} \mathrm{P}_{0}^{\mathrm{o}}-{ }^{3} \mathrm{D}_{1}$ & 2326.05 & 1 & 39.23 & -22.26 \\
\hline & & & 2 & 39.08 & -27.12 \\
\hline & & & 3 & 36.02 & -28.88 \\
\hline & & & 5 & 31.30 & -27.32 \\
\hline & & & 10 & 30.80 & -22.88 \\
\hline & & & 16 & 29.03 & -20.04 \\
\hline \multirow[t]{6}{*}{$5 \mathrm{~d}^{10} 6 \mathrm{~s}\left({ }^{2} \mathrm{~S}\right) 7 \mathrm{p}-5 \mathrm{~d}^{10} 6 \mathrm{~s}\left({ }^{2} \mathrm{~S}\right) 7 \mathrm{~d}$} & ${ }^{3} \mathrm{P}_{1}^{\mathrm{o}}-{ }^{3} \mathrm{D}_{1}$ & 2326.89 & 1 & 51.05 & -23.56 \\
\hline & & & 2 & 48.06 & -27.43 \\
\hline & & & 3 & 42.26 & -30.24 \\
\hline & & & 5 & 35.34 & -24.91 \\
\hline & & & 10 & 36.92 & -21.46 \\
\hline & & & 16 & 35.74 & -19.18 \\
\hline \multirow[t]{6}{*}{$5 d^{10} 6 s\left({ }^{2} S\right) 7 p-5 d^{10} 6 s\left({ }^{2} S\right) 7 d$} & ${ }^{3} \mathrm{P}_{1}^{\mathrm{o}}-{ }^{3} \mathrm{D}_{2}$ & 2311.83 & 1 & 74.30 & -39.00 \\
\hline & & & 2 & 71.68 & -45.72 \\
\hline & & & 3 & 65.31 & -48.36 \\
\hline & & & 5 & 55.55 & -43.46 \\
\hline & & & 10 & 55.60 & -36.63 \\
\hline & & & 16 & 52.86 & -32.26 \\
\hline \multirow[t]{6}{*}{$5 d^{9} 6 s^{2} 6 p-5 d^{10} 6 s\left({ }^{2} s\right) 7 d$} & $14_{2}^{\mathrm{o}}-{ }^{3} \mathrm{D}_{3}$ & $2627.91^{*}$ & 1 & 149.67 & -86.24 \\
\hline & & & 2 & 139.65 & -94.84 \\
\hline & & & 3 & 127.24 & -96.53 \\
\hline & & & 5 & 105.57 & -89.79 \\
\hline & & & 10 & 105.07 & -74.88 \\
\hline & & & 16 & 99.60 & -65.50 \\
\hline \multirow[t]{6}{*}{$5 \mathrm{~d}^{10} 6 \mathrm{~s}\left({ }^{2} \mathrm{~S}\right) 7 \mathrm{p}-5 \mathrm{~d}^{10} 6 \mathrm{~s}\left({ }^{2} \mathrm{~S}\right) 7 \mathrm{~d}$} & ${ }^{3} \mathrm{P}_{2}^{\mathrm{o}}-{ }^{3} \mathrm{D}_{3}$ & 2772.92 & 1 & 132.22 & -88.35 \\
\hline & & & 2 & 131.14 & -100.16 \\
\hline & & & 3 & 125.09 & -99.76 \\
\hline & & & 5 & 108.98 & -99.72 \\
\hline & & & 10 & 100.93 & -81.29 \\
\hline & & & 16 & 92.63 & -70.04 \\
\hline \multirow[t]{6}{*}{$5 \mathrm{~d}^{10} 6 \mathrm{~s}\left({ }^{2} \mathrm{~S}\right) 5 \mathrm{f}-5 \mathrm{~d}^{10} 6 \mathrm{~s}\left({ }^{2} \mathrm{~S}\right) 7 \mathrm{~d}$} & ${ }^{3} \mathrm{~F}_{4}^{\mathrm{o}}-{ }^{3} \mathrm{D}_{3}$ & $3027.28^{*}$ & 1 & 164.48 & -100.45 \\
\hline & & & 2 & 161.18 & -115.95 \\
\hline & & & 3 & 153.07 & -116.10 \\
\hline & & & 5 & 131.58 & -117.78 \\
\hline & & & 10 & 123.57 & -94.66 \\
\hline & & & 16 & 114.11 & -80.93 \\
\hline \multirow[t]{6}{*}{$5 d^{10} 6 s\left({ }^{2} S\right) 7 p-5 d^{10} 6 s\left({ }^{2} S\right) 7 d$} & ${ }^{3} \mathrm{P}_{1}^{\mathrm{o}}-{ }^{1} \mathrm{D}_{2}$ & $2214.46^{*}$ & 1 & 81.15 & -50.38 \\
\hline & & & 2 & 75.01 & -52.14 \\
\hline & & & 3 & 67.39 & -52.73 \\
\hline & & & 5 & 56.69 & -46.39 \\
\hline & & & 10 & 54.79 & -38.45 \\
\hline & & & 16 & 51.32 & -33.57 \\
\hline \multirow[t]{6}{*}{$5 d^{9} 6 s^{2} 6 p-5 d^{10} 6 s\left({ }^{2} s\right) 7 d$} & $13_{1}^{\mathrm{o}}-{ }^{1} \mathrm{D}_{2}$ & $2402.06^{*}$ & 1 & 89.89 & -59.10 \\
\hline & & & 2 & 84.42 & -61.12 \\
\hline & & & 3 & 76.49 & -61.52 \\
\hline & & & 5 & 65.31 & -55.94 \\
\hline & & & 10 & 62.19 & -45.91 \\
\hline & & & 16 & 57.84 & -39.84 \\
\hline \multirow[t]{6}{*}{$5 \mathrm{~d}^{10} 6 \mathrm{~s}\left({ }^{2} \mathrm{~S}\right) 7 \mathrm{p}-5 \mathrm{~d}^{10} 6 \mathrm{~s}\left({ }^{2} \mathrm{~S}\right) 7 \mathrm{~d}$} & ${ }^{1} \mathrm{P}_{1}^{\mathrm{o}}-{ }^{1} \mathrm{D}_{2}$ & 2642.36 & 1 & 97.39 & -69.46 \\
\hline & & & 2 & 93.10 & -73.50 \\
\hline & & & 3 & 87.73 & -71.52 \\
\hline & & & 5 & 75.03 & -69.80 \\
\hline & & & 10 & 68.92 & -56.87 \\
\hline & & & 16 & 63.00 & -49.05 \\
\hline \multirow{6}{*}{$5 d^{10} 6 s\left({ }^{2} S\right) 5 f-5 d^{10} 6 s\left({ }^{2} S\right) 7 d$} & ${ }^{3} \mathrm{~F}_{3}^{\mathrm{o}}-{ }^{1} \mathrm{D}_{2}$ & 2793.06 & 1 & 116.67 & -69.24 \\
\hline & & & 2 & 108.24 & -77.54 \\
\hline & & & 3 & 102.93 & -74.70 \\
\hline & & & 5 & 88.95 & -72.63 \\
\hline & & & 10 & 82.29 & -58.02 \\
\hline & & & 16 & 75.84 & -49.08 \\
\hline
\end{tabular}

Notes. ${ }^{*}$ A positive shift is red.

${ }^{a}$ Moore (1958). 
Table 5. Bi Iv $5 \mathrm{~d}^{10} 6 \mathrm{~s}\left({ }^{2} \mathrm{~S}\right) 5 \mathrm{f}$ line widths (FWHM), $\omega(\mathrm{pm})$, and shifts, $d(\mathrm{pm})$, normalized to $N e=10^{17}$ $\mathrm{cm}^{-3}$.

\begin{tabular}{|c|c|c|c|c|c|}
\hline Transition array & Multiplet & $\lambda(\AA)^{a}$ & $T\left(10^{4} \mathrm{~K}\right)$ & $\omega(\mathrm{pm})$ & $d(\mathrm{pm})$ \\
\hline \multirow[t]{6}{*}{$5 d^{10} 6 s\left({ }^{2} S\right) 6 d-5 d^{10} 6 s\left({ }^{2} S\right) 5 f$} & ${ }^{3} \mathrm{D}_{2}-{ }^{3} \mathrm{~F}_{3}^{\mathrm{o}}$ & 2768.09 & 1 & 30.47 & -4.90 \\
\hline & & & 2 & 20.08 & -4.76 \\
\hline & & & 3 & 16.31 & -0.71 \\
\hline & & & 5 & 12.52 & 1.69 \\
\hline & & & 10 & 15.70 & -1.36 \\
\hline & & & 16 & 16.66 & -2.55 \\
\hline \multirow[t]{6}{*}{$5 d^{10} 6 s\left({ }^{2} S\right) 6 d-5 d^{10} 6 s\left({ }^{2} S\right) 5 f$} & ${ }^{3} \mathrm{D}_{3}-{ }^{3} \mathrm{~F}_{3}^{\mathrm{o}}$ & 2925.17 & 1 & 42.17 & -11.24 \\
\hline & & & 2 & 28.23 & -9.45 \\
\hline & & & 3 & 18.21 & 0.58 \\
\hline & & & 5 & 16.63 & 0.30 \\
\hline & & & 10 & 20.15 & -5.70 \\
\hline & & & 16 & 22.76 & -6.33 \\
\hline \multirow[t]{6}{*}{$5 \mathrm{~d}^{10} 6 \mathrm{p}^{2}-5 \mathrm{~d}^{10} 6 \mathrm{~s}\left({ }^{2} \mathrm{~S}\right) 5 \mathrm{f}$} & ${ }^{3} \mathrm{P}_{2}-{ }^{3} \mathrm{~F}_{3}^{\mathrm{o}}$ & $3172.49^{*}$ & 1 & 26.80 & 5.85 \\
\hline & & & 2 & 17.14 & 2.32 \\
\hline & & & 3 & 15.24 & 4.71 \\
\hline & & & 5 & 12.25 & 5.99 \\
\hline & & & 10 & 14.43 & 4.18 \\
\hline & & & 16 & 15.09 & 3.59 \\
\hline \multirow[t]{6}{*}{$5 d^{10} 6 s\left({ }^{2} S\right) 6 d-5 d^{10} 6 s\left({ }^{2} S\right) 5 f$} & ${ }^{3} \mathrm{D}_{1}-{ }^{3} \mathrm{~F}_{2}^{\mathrm{o}}$ & 2630.13 & 1 & 11.54 & -5.89 \\
\hline & & & 2 & 8.16 & -4.17 \\
\hline & & & 3 & 5.11 & -1.84 \\
\hline & & & 5 & 3.66 & -1.87 \\
\hline & & & 10 & 5.28 & -3.96 \\
\hline & & & 16 & 5.62 & -3.96 \\
\hline \multirow[t]{6}{*}{$5 d^{10} 6 s\left({ }^{2} S\right) 6 d-5 d^{10} 6 s\left({ }^{2} S\right) 5 f$} & ${ }^{3} \mathrm{D}_{2}-{ }^{3} \mathrm{~F}_{2}^{\mathrm{o}}$ & 2677.88 & 1 & 18.36 & -10.45 \\
\hline & & & 2 & 12.91 & -7.31 \\
\hline & & & 3 & 8.65 & -4.08 \\
\hline & & & 5 & 6.03 & -3.26 \\
\hline & & & 10 & 8.34 & -5.80 \\
\hline & & & 16 & 8.92 & -6.92 \\
\hline \multirow[t]{6}{*}{$5 d^{10} 6 s\left({ }^{2} S\right) 6 d-5 d^{10} 6 s\left({ }^{2} S\right) 5 f$} & ${ }^{3} \mathrm{D}_{3}-{ }^{3} \mathrm{~F}_{4}^{\mathrm{o}}$ & 2786.45 & 1 & 34.44 & -13.11 \\
\hline & & & 2 & 24.31 & -9.23 \\
\hline & & & 3 & 13.89 & -1.58 \\
\hline & & & 5 & 10.77 & -4.14 \\
\hline & & & 10 & 14.83 & -8.42 \\
\hline & & & 16 & 17.30 & -8.78 \\
\hline \multirow[t]{6}{*}{$5 d^{10} 6 s\left({ }^{2} S\right) 6 d-5 d^{10} 6 s\left({ }^{2} S\right) 5 f$} & ${ }^{1} \mathrm{D}_{2}-{ }^{1} \mathrm{~F}_{3}^{\mathrm{o}}$ & 1909.93 & 1 & 1.90 & -1.42 \\
\hline & & & 2 & 1.34 & -1.00 \\
\hline & & & 3 & 1.10 & -0.82 \\
\hline & & & 5 & 0.68 & -0.47 \\
\hline & & & 10 & 0.57 & -0.47 \\
\hline & & & 16 & 0.74 & -0.61 \\
\hline \multirow[t]{6}{*}{$5 d^{10} 6 p^{2}-5 d^{10} 6 s\left({ }^{2} S\right) 5 f$} & ${ }^{3} \mathrm{P}_{2}-{ }^{1} \mathrm{~F}_{3}^{\mathrm{o}}$ & 2933.93 & 1 & 8.42 & -2.86 \\
\hline & & & 2 & 5.95 & -2.03 \\
\hline & & & 3 & 4.19 & -0.99 \\
\hline & & & 5 & 3.15 & -0.70 \\
\hline & & & 10 & 3.66 & -2.13 \\
\hline & & & 16 & 3.69 & -2.72 \\
\hline \multirow[t]{6}{*}{$5 d^{10} 6 p^{2}-5 d^{10} 6 s\left({ }^{2} S\right) 5 f$} & ${ }^{1} \mathrm{D}_{2}-{ }^{1} \mathrm{~F}_{3}^{\mathrm{o}}$ & 5349.02 & 1 & 52.28 & -15.96 \\
\hline & & & 2 & 38.27 & -12.62 \\
\hline & & & 3 & 41.02 & -20.05 \\
\hline & & & 5 & 41.23 & -25.09 \\
\hline & & & 10 & 38.35 & -26.75 \\
\hline & & & 16 & 34.45 & -28.67 \\
\hline
\end{tabular}

Notes. ${ }^{*}$ A positive shift is red.

${ }^{a}$ Moore (1958).

that are excluded from the fitting process, which is the procedure suggested by COWAN. Our parameters differ slightly from the parameters obtained by Martin et al. (1972), which focused on the best possible adjustment of energy levels (their calculated lev- els differ by less than 0.5 per cent of the experimental ones) and which could lead us to transition probabilities excessively influenced by the mixtures of configurations, and in some case forced to obtain the adjustment desired by these authors. The parameters 

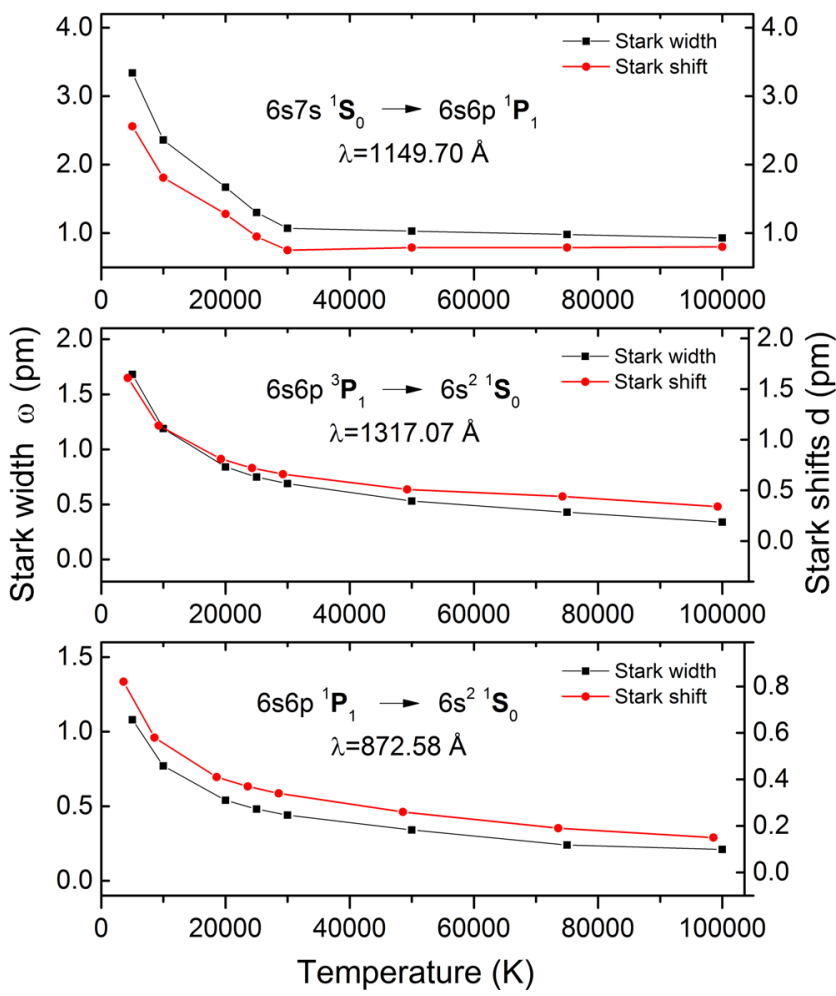

Figure 3. Calculated Stark width FWHM $[\omega(\mathrm{pm})]$ and shift $[d(\mathrm{pm})]$ at an electron density of $10^{17} \mathrm{~cm}^{-3}$ versus temperature for several spectral lines of Bi Iv.

obtained in this work after the adjustment process and that do not differ by more than 10 per cent of the ab initio values are available on request.

\section{RESULTS AND DISCUSSION}

Results obtained in this work for the lifetimes of 12 levels of Bi IV which have experimental values available in the bibliography and its comparison with these experimental values are presented in Table 1. The ratio between experimental values and our theoretical values is displayed in Fig. 2. Fig. 2 shows that that our values are in good agreement with the experimental results even in the worst case presented, the value for the $5 \mathrm{~d}^{10} 6 \mathrm{~s}\left({ }^{2} \mathrm{~S}\right) 6 \mathrm{~d}{ }^{1} \mathrm{D}_{2}$ level that was obtained through the measurement with the $1437.6 \AA$ spectral line of $\mathrm{Bi}$ IV very close to the $1436.8 \AA$ spectral line of $\mathrm{Bi}$ II. So we think that our matrix elements calculated in this work are precise enough to calculate the Stark broadening parameters.

Our results for the Stark broadening parameters are shown in Tables 2-5 for an electronic density of $10^{17} \mathrm{~cm}^{-3}$ and temperatures ranging from 10000 to $160000 \mathrm{~K}$ except for three lines, which are presented below in Fig. 3, and are presented for a range from 5000 to $160000 \mathrm{~K}$. The transition array, the multiplet and the wavelengths (in $\AA$ ) for each studied transition are displayed in Columns 1-3, respectively. In Columns 5 and 6, our theoretical Stark line width and line shift (a negative shift is blue) are displayed (in pm). Spectral lines marked with * are lines not collected by NIST (Kramida, Ralchenko \& Reader 2013) but were identified by McLay in 1933. Following Griem's comments, a difference of a factor of 1.5 can be expected between the experimental values and our calculations. This is due to the experimental problem of precision in the measurement of electron densities and temperature. Also in our case the energy

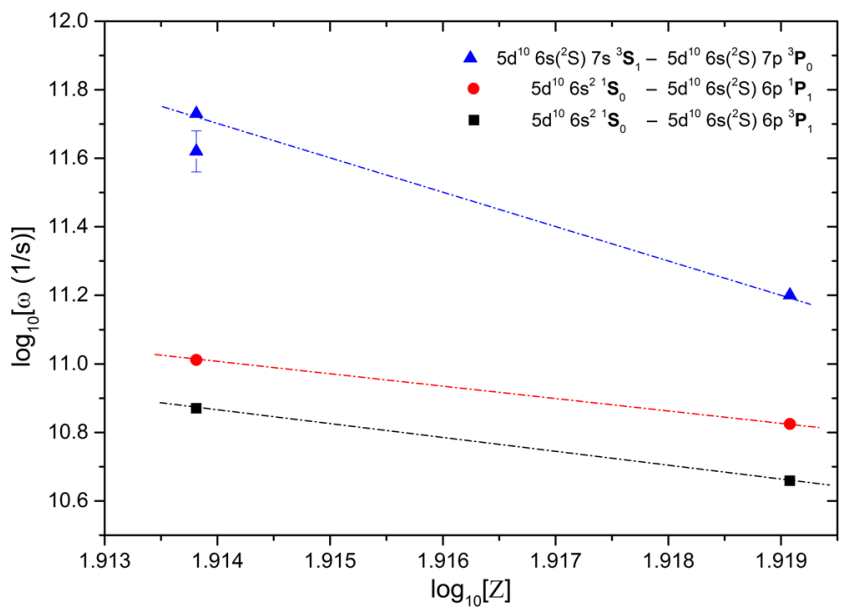

Figure 4. Stark width FWHM [ $\omega$ (in frequency units)] of three transitions at an electron density of $10^{17} \mathrm{~cm}^{-3}$ in $\mathrm{Pb}$ III and $\mathrm{Bi}$ IV versus the ion charge (number Z): $\square$ and $\bullet$, theoretical values at a temperature of $20000 \mathrm{~K}$ (this work and Zanón et al. 2010); $\mathbf{\Delta}$, theoretical values at a temperature of $21400 \mathrm{~K}$ (this work and Zanón et al. 2010) and the experimental value (data with error bars) at a temperature of $21400 \mathrm{~K}$ (Alonso-Medina 2011).

differences of the perturbing levels can reach $15 \mathrm{eV}$, close to the limits of validity of the used Gaunt factors.

The dependence between the temperature and our widths FWHM $[\omega(\mathrm{pm})]$, as well as the dependence between the temperature and our Stark line shifts $[d(\mathrm{pm})]$, is displayed, respectively, in Fig. 3. 1149.70, 1317.07 and 872.58 § Bi Iv lines were selected. A comparison of measured and calculated Stark width of $\mathrm{Pb}$ III, obtained by these authors, Zanón et al. (2010) and Alonso-Medina (2011), with calculated Stark width of Bi IV (same isoelectronic sequence) in this work, is displayed for three transitions in Fig. 4. Although there are only two values in each curve, a dependence of the Stark widths compatible with $Z^{-2}$ can be observed.

In conclusion, in this work, we have calculated the Stark widths and shifts of $64 \mathrm{Bi}$ IV spectral lines. The semi-empirical model of Griem, and the COWAN code modified to include CP effects to obtain the necessary matrix elements, has been used. These values are the first reported in the literature. The dependence of these values with the temperature and with the charge number $Z$ has been studied.

\section{ACKNOWLEDGEMENTS}

This work was financially supported by the Spanish Ministry of Science and Technology (DGI project MAT-2015-63974-C4-2-R).

\section{REFERENCES}

Alonso-Medina A., 2010, J. Quant. Spectrosc. Radiat. Transfer, 111, 2043 Alonso-Medina A., 2011, Spectrochim. Acta B 66, 439

Alonso-Medina A., Colón C., 2007, A\&A, 466, 399

Alonso-Medina A., Colón C., 2014, MNRAS, 445, 1567

Alonso-Medina A., Herrán C., 1996, Phys. Scr., 54, 332

Alonso-Medina A., Colón C., Zanón A., 2008, MNRAS, 385, 261

Alonso-Medina A., Colón C., Zanón A., 2009, MNRAS, 395, 567

Alonso-Medina A., Colón C., Montero J. L., Nation L., 2010, MNRAS, 401, 1080

Andersen T., Kirkegård Nielsen A., Sørensen G., 1972, Phys. Scr., 6, 122

Ansbaher W., Pinnington E. H., Tauheed A., Kernahan J. A., 1989, Phys. Scr., 40,454 
Banahan C., McGuinness C., Costello J. T., Kilbane D., Mosnier J.-P., Kennedy E. T., O'Sullivan G., van Kampen P., 2008, J. Phys. B: At. Mol. Opt. Phys., 41, 205001

Baranger M., 1958, Phys. Rev., 112, 855

Colón C., Alonso-Medina A., 2000, Phys. Scr., 62, 132

Colón C., Alonso-Medina A., 2002, J. Phys. B: At. Mol. Opt. Phys., 35, 1143

Colón C., Alonso-Medina A., Herran-Mertínez C., 1999, J. Phys. B: At. Mol. Opt. Phys., 32, 3887

Colón C., Moreno-Díaz C., Alonso-Medina A., 2013, MNRAS, 435, 1749

Cowan R. D., 1981, The Theory of Atomic Structure and Spectra. University of California Press, Berkeley, USA

Curtis L. J., Irving R. E., Henderson M., 2001, Phys. Rev. A, 63, 042502

de Andrés-García I., Alonso-Medina A., Colón C., 2016, MNRAS, 455, 1145

Dolk L., Litzén U., Wahlgren G. M., 2002, A\&A, 388, 692

Griem H. R., 1968, Phys. Rev., 165, 258

Hameed S., 1972, J. Phys. B: At. Mol. Opt. Phys., 5, 746

Kramida A., Ralchenko Y. U., Reader J.; NIST ASD Team, 2013, NIST Atomic Spectra Database (v.5.3), http://physics.nist.gov/asd Martin W. C., Sugar J., Tech J. L., 1972, Phys. Rev. A, 6, 2022

McLay A. B., Crawford M. F., 1933, Phys. Rev., 44, 986
Migdalek J., Baylis W. E., 1978, J. Phys. B: At. Mol. Opt. Phys., 11, L497 Migdalek J., Baylis W. E., 1985, J. Phys. B: At. Mol. Opt. Phys., 18, 1533

Moore C. E., 1958, Atomic Energy Levels, NBS Circular 467, Vol. III. U.S. Government Printing Office, Washington, DC, p. 213

Moreno-Díaz C., Alonso-Medina A., Colón C., 2014, Phys. Scr., 89, 115401

Pinnington E. H., Ansbacher W., Kernahan J. A., Ge Z.-Q., Inamdar A. S., 1988, Nucl. Instrum. Methods Phys. Res. B, 31, 206

Ryabtsev A. N., Churilov S. S., Joshi Y. N., 2000, Opt. Spectrosc., 88(3), 360

Seaton M. J., 1962, in Bates, D. R., ed., Atomic and Molecular Processes. Academic Press, New York

Van Regemorter H., 1962, ApJ, 136, 906

Wahlgren G. M., Brage T., Brandt J. C., Fleming J., Johansson S., Leckrone D. S., Proffitt C. R., Reader J., Sansonetti C. J., 2001, Astrophys. J., 551, 520

Zanón A., Alonso-Medina A., Colón C., 2010, Int. Rev. At. Mol. Phys., 1, 1

Zorenko Y. et al., 2009, J. Phys. B: App. Phys., 42, 075501

Zorenko Y. et al., 2014, J. Lumin., 154, 525

This paper has been typeset from a $\mathrm{T}_{\mathrm{E}} \mathrm{X} / \mathrm{LT} \mathrm{E} \mathrm{X}$ file prepared by the author. 